NASA Technical Memorandum 86865

\title{
Computational Thermo-Fluid Dynamics Contributions to Advanced Gas Turbine Engine Design
}

Robert W. Graham, John J. Adamczyk, and Harold E. Rohlik Lewis Research Center Cleveland, Ohio

Prepared for the

Twenty-third Aerospace Sciences Meeting sponsored by the American Institute of Aeronautics and Astronautics Reno, Nevada, January 14-17, 1985

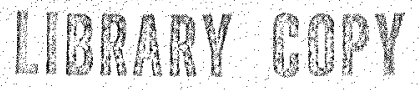

ANOL W RESWRCH OEMTH LOWhW, WhS HMWTON. VROM

\section{NNSA}




\title{
COMPUTATIONAL. THERMO-FLUID DYNAMICS CONTRIBUTIONS TO ADVANCED GAS
} TURBINE ENGINE DESIGN

\author{
Robert W. Graham, John J. Adamczyk, and Harold E. Rohlik \\ National Aeronautics and Space Administration \\ Lewis Research Center \\ Cleveland, Ohio 44135
}

\section{Abstract}

The early history of design practice for the aircraft gas turbine engine can be characterized as the application of empiricism founded on actual test experience. Trial and error processes gave the designer the needed information to modify or redesign the principal components for more stringent operating conditions but the changes between engine models were conservatively small. In many cases, this approach was both extremely costly and time consuming. A sizeable fraction of the cost of marketing a new engine went into equipment modification and the iterative substitution of design changes. Aircraft gas turbine engines $* \quad$ being envisioned for the last decade of this cenI tury and the early decades of the next century will operate at higher pressures and temperatures. Tolerances and clearances will be tighter than current practices. Design practice will rely heavily on computational codes to predict gas flows and thermal conditions throughout the flow path of the engine so that iterative changes in the development of the engine will be minimized. The introduction of more computational methods into the design process will enable bolder changes in the evolution of new engines. Improved cycle efficiency, quieter operation and more reliable performance will be among the benefits derived from this design practice.

\section{Introduction}

Over the past 40 years the gas turbine power plant for aircraft propulsion has made remarkable progress. However, this progress has not been made without considerable difficulty. The learning process in design has often been an arduous, frustrating experience marked with failure as well as achievement. During most of the evolutionary history of the aircraft gas turbine, the designer found himself dealing with technical decisions that were beyond the state of available technological knowledge or experience. He was compelled to take risks of innovation and extrapolated technical practice to meet the operating demands of the engine. In the early days there were few analytical tools to bridge the gap between established practice and the design demands of the gas turbine engine. Trial and error was one of the practical ways of overcoming the gap. This process eventually become a development testing program in which new concepts were tried and modified until they worked and proved themselves. While the process was reasonably successful, it became increasingly apparent that the iteration process between fabrication and test was becoming prohibitively costly and time consuming.

In conjunction with experimental development, fundamental research activity expanded which provided a foundation of information or data for improved design correlations or analysis. This research encompassed a broad spectrum of disciplines including fluid mechanics, aerodynamics, heat transfer, thermodynamics, structural mechanics and materials. The engine companies, universities and government laboratories became joint participants in this research endeavor. The advent of the electronic computer and the electronic acquisition and recording of data has had a major influence on how fundamental research information is utilized in the design of advanced gas turbines. There is a definite movement toward reliance on computational methods to establish the design of advanced engines. The mechanical calculator and slide rule have given way to the large storage computers; the manometer tube banks and strip charts have been replaced with transducers and CRT's.

It is still too early to predict what potential capability this new computational tool will make possible. Hopefully, it will enhance innovative design, improve the certainity of predictions and minimize costly design changes during the buildup of a new engine.

In this paper the authors will trace the design practices for the gas turbine throughout its history with particular emphasis on the calculational or analytical methods. With such a background and a picture of the current situation, we will make an assessment of the future impact of computational fluid mechanics on future gas turbine design. Three principal components of the gas turbine engine will be considered: namely, the compressor, the combustor and the turbine.

\section{Components of the Gas Turbine}

\section{The Compressor Early Design Approaches}

In the beginnings of the gas turbine power plant, the performance of the compressor was probably the most crucial design issue of all the components. It was the inefficiency of the available compressors that delayed the introduction of the gas turbine as a viable power plant. Advances in the design and production of superchargers for aircraft reciprocating engines contributed significantly to the improvement of compressor performance. These initial improvements came about from empirical information derived from development experience. Along with the improved compressor machinery, more systemmatic design methods were also evolving which enabled subsequent improvements in later models. This "bootstrap" process advanced the design methodology dramatically in the first generations of the aircraft gas turbine.

The earliest U.S. compressor used in an aircraft gas turbine was a centrifugal type. For the larger engines, the centrifugal was quickly replaced with the axial flow because of its smaller frontal profile and multistaging possibilities. The axial-flow has remained the preferred compressor configuration for the large engines used in civil transports and most military aircraft, except for helicopters. The airfoil shapes of the 
blades and vanes of the axial flow machine lend themselves to airfoil wing theory in the design practice. The "blade element" design approach resulted from this association. In this design approach, shown schematically in Fig. 1, each blade row was considered to be made up of a finite number of elements which are radially stacked. Velocity diagrams for the leading and trailing edges of each element were selected to give the proper flow turning and pass the required quantity of air. A blockage correction was applied to the flow area to account for the presence of the blades. An airfoil shape was selected from a family of known airfoils (generally the NACA types) which would accommodate the turning and velocity diagram specifications. Demands for increased pressure rise per stage in more advanced machines called for maximized loading or turning in each blade row. This demand necessitated loading and loss information obtained in experiments with stationary cascades of blades which simulated the compressor stages.

In addition to the cascade approach, the performance of single stage research compressors was used to ascertain loading limits and losses. Analyses of these types of data from both cascades and single stage research compressors were utilized in establishing empirical guides for loading limits and losses for individual blade rows.

The early multistage design procedure amounted to extensions of the single stage procedure. One method was called "stage stacking." This empirical method made use of dimensionless pressure and flow coefficient maps of each single stage similar to those shown in $\mathrm{Fig}$. 2. By matching these stage performance maps with the flow angles of the velocity diagrams, an initial multistage design resulted. Performance checks of compressors designed in this fashion revealed severe limitations of the approach, particularly in integrating the secondary flow losses and the three-dimensional reality of the flow through the machine. The relative success of the method depended heavily on how well the dimensionless performance maps of each stage were known.

Comprehensive descriptions of the design procedures and pertinent comments about the history of compressor design are documented in reference 1. Early on in the design history, the major limitations of the practice were identified. Analytical approaches as well as experimentallyfounded empiricisms were initiated to overcome these limitations. Some of the analytical approaches predate the general availability of the electronic computer. For example, the threedimensional theory ${ }^{2}$ appeared in the early $50^{\prime} \mathrm{s}$. This reference was a major contributor to the pseudo-three dimensional numerical techniques that followed in the $60^{\prime} \mathrm{s}$ and $70^{\prime} \mathrm{s}$ in which "flow through" or "flow path" computational methods were developed. The flow path approach yields meanline information along prescribed flow surfaces. The equation set includes the momentum equation, the continuity equation, the energy equation and the equation of state.

\section{Current Design Practice}

At this writing, the prevalent analytical methods used by the industry in design are the above pseudo-three dimensional types in which the calculations are carried out on two orthogonal surfaces within a typical blade passage (see Fig. $3)$. The solutions are carried out for inviscid, steady flow. Viscous effects are included by boundary layer computations along the wall and blade surfaces.

\section{Future Design Approaches}

Under development are numerical codes for three dimensional flows that apply to steady, inviscid and viscid conditions. As these codes become verified, they will replace the codes now in use which approximate the three dimensional geometry by gas path solutions on meridional and blade-to-blade surfaces. It is hoped the truly three-dimensional analyses will enable lessempirical loss estimates and also provide prediction of the secondary flows. These three dimensional codes are premised on the assumption of steady flow. The inclusion of the grossly unsteady features of the real flow is an issue which still needs to be addressed. A proposed scheme for doing this will be described in the next section of this paper.

Comparing the new design procedure for compressors with the initial blade element approaches, it is apparent that more information about the internal flow characteristics of blade rows is being incorporated. The early versions depended on information pertaining to the inlet and discharge stations of the blade row only. The new computational ability and also the availability of nonintrusive flow measurements within the blade rows have made this major change possible. This comment pertains to turbine design as well as to the compressor.

\section{An Analytical Approach for Multistage}

\section{Turbomachinery}

In the discussion on compressor design analysis it was mentioned that the current aerodynamic design of turbomachines is generally based on a combined through-flow and blade-to-blade analys is to simulate three dimensional flow. Such an analysis described the average passage flow field which is considered to be steady and is periodic from passage to passage. A more rigorous method has been proposed ${ }^{3}$ in which the equations are truly three-dimensional and methods for averaging the passage flows are suggested.

The derivation of the steady threedimensional average-passage equation system begins by ensemble averaging the three-dimensional Navier-Stokes equations. This is done to eliminate the need to resolve in detail the structure of turbulent flows. The global effects of turbulence on the deterministic flow field are accounted for by means of a Reynolds stress and a scalar flux tensor. The resulting system of equations, often referred to as the Reynolds-averaged equations, are then averaged in time at every point in space to remove unsteady time scales which are on the order of the period of shaft rotation or greater. The global effect of the organized (as opposed to random) blade-to-blade unsteady flow structure manifests itself in the resulting equation system as body forces, energy sources, momentum and energy temporal mixing correlations. For a single-stage machine subjected to an axisymmetric inlet and exit condition this equation set 
describes the three-dimensional average-passage flow field associated with either the rotor or stator blade row.

In regard to multistage machines the ensemble time-averaged equations govern, in general, a flow field which varies from passage to passage around a given blade row. To extract the average-passage equations from this equation system, they must be averaged on a passage-to-passage basis. The mathematical operator which accomplishes this task averages out the details of the passage-to-passage variation in the flow field. However the global effect on the average-passage flow field is not eliminated. Its existence is accounted for through body forces, energy sources, momentum, and energy spatial mixing correlations.

A set of average-passage flow equations contain correlations which arise from turbulence, unsteady organized flow, and passage-to-passage flow variations. These correlations are generic to this equation system, just as the Reynolds stress tensor is generic to the Reynolds-averaged Navier-Stokes equations. They serve as the mechanism by which the passage-average flow field is mixed out in the multistage environment. The need for accounting for this mixing process in turbomachinery flow analyses was recently documented. 4

In Ref. 3, two reduced forms of the averagepassage flow equations are developed. In deriving the first set of equations it is assumed that the body forces and energy sources generated by the presence of neighboring blade rows can be evaluated using a through-flow blade-to-blade analysis. It is further assumed that the mixing correlations can be modeled in both the through-flow blade-toblade analysis and the average-passage system according to the analysis presented. 4 The resulting set of equations describe a threedimensional flow field whose axisymmetric component is equal to that predicted by the through-flow blade-to-blade model. In the second approach, an average-passage equation system is developed for each blade row in the machine. The two sets of equations are coupled through common expressions for the mixing correlations, the body forces and the energy sources. Thus they must be solved simultaneously. The closure problem associated with this formulation requires the development of models which globally describe the mixing produced by the coherent spatial and temporal nonuniform flow in addition to models for the Reynolds stress and associated turbulent correlations.

An alternative approach to the closure problem is to consider separately the mixing stress, the body forces and energy sources not associated with the flow turbulence. The contribution of the coherent velocity field to the mixing correlations can be decomposed into two components - an incident gust and a velocity field which is the source of the gust.

The above-mentioned closure suggestions have not been evaluated. A considerable effort in numerical analysis will be necessary for this task. An analytical effort alone will not be sufficient because of the complexity of the problem. Detailed measurements of the fluctuating airloads and the aerodynamic noise associated with multi-stage blade rows wi11 be necessary to support the computational effort. Certainly, the verification of the closure approaches will require a major experimental test program as well.

The Combustor Early Design Practice

The combustor appears to be one of the most mechanically-simplistic components of the gas turbine engine. Its outward simplicity is deceiving, however, because it houses a complex aerodynamic and chemical reaction processer that challenge the ingenuity of the designer (see Fig. 4). Those who designed the first combustors for aircraft gas turbines had very little prior engineering experience to build on. The principles of flame combustion had been a scientific curiosity from the early experiments of Bunsen but making an energyintensive reactor work was a challenging new technology. This challenge incited a major effort in combustion research for the conditions encountered in the gas turbine combustor.

The principal functions of a combustor are to accept the high pressure air from the compressor discharge, reduce the airflow velocity to values approximating flame speeds, distribute and mix fuel in that air stream and homogenize the combustion gases into a uniform or prescribed temperature profile at the combustor discharge. Research and development efforts were initiated that examined elements of the process that occur within the combustor. Among the principal topics were the following.

1. the diffusion process in the combustor inlet.

2. the fuel spray patterns of the fuel injectors.

3. the ignition of the fuel/air mixture.

4. the mixing and diffusion of the combustion reactants.

5. dilution of the combustion products.

The major overall objective of these studies was to aid the designer in providing satisfactory mixing, stable flow and burning while maintaining minimal pressure drop through the combustor. Much of this research was experimental and provided data sets or correlations that could be applied to design practice. Most of these were empirical but some theoretical effort to understand flammability, flame propagation, diffusion and other phenomena were being pursued along with the experiments. 6

During the decades of the $50^{\prime} \mathrm{s}, 60^{\prime} \mathrm{s}$ and even some of the $70^{\prime} s$, combustor design was more of an art than a science. Combustor hardware was built, tested and modified until it performed in conformity to the constraints of specifications. In the course of developing a satisfactory combustor, some of the changes were easily performed such as adding new holes in the combustor liners or the introduction of new vane geometrics to channel the cooling air. ${ }^{7}$ Sometimes more major changes were needed that required complete revision of the combustor configuration. The designer was sent back to the drawing board. Modification of a combustor design, to any degree, is an expensive, time consuming process. As labor and material 
costs soared during the late seventies, it became increasingly evident that the cut-and-try-type process of development was becoming prohibitively costly. Improved design techniques were needed that were founded on fundamental knowledge of aerodynamics, mixing, combustion and dilution. Analysis must become the principal component of the design method. Design changes are rendered more economically on paper than in sheet metal.

\section{A New Combustor Design Philosophy}

Over the past decade significant progress has been made in modeling the processes that go on in a combustor. The modeling has involved definitive experiments to elucidate the processes and computational methods which simulate the betterunderstood physical and thermodynamic processes. For example, one of the areas has been spray production. Laser diagnostic systems have been employed to characterize the drop sizes, distribution and velocities. Compared to the early visual and wax-solidification methods used to get Sauter mean diameters as characterization of the spray, these new laser measurements are a major step forward.

Another important development in combustion research is the ability to predict the combustion flow field. Reference 8 reports on a method that models combustion in a two-dimensional combustor geometry. The foundation of the analysis is a random vortex method developed at the University of California-Berkeley. The method models turbulence from first principles, tracking the vorticity and predicting the interaction of the vorticity with the bulk flow. The random vortex method has been incorporated in a computer program called MIMOC (Modeling the Interface Motion of Combustion,) which can be used to predict the reacting flow field behind a rearward facing step. This computer program evolved from a cooperative effort between University of California Berkeley and the Lewis Research Center. The output of this program can be coupled to computer graphics to render images of the combustion flow field. Figure 5 shows the images of the combustion field in three time steps downstream of a step in a two-dimensional channel. These computer-generated images compare favorably with Schlieren images of real combustion in a similar geometry.

Reference 8 represents one effort to model a particular turbulent flow field. Currently there is an extensive effort within the computational fluid mechanics community in the modeling of turbulent flows. The general progress in this area will have important impact on the modeling of combustion flows. In this paper we cannot attempt to review this extensive body of work on turbulence modeling and interpret the progress being made as it effects combustion modeling. Another single example of a continuing effort in this area is the turbulence modeling effort being sponsored by the Naval Research Laboratory. ${ }^{9}$ In this reference computational studies of unstable two-dimensional shear flows transitioning to turbulence have been reported. The first versions of the model include only convective terms but continuing efforts will include the effects of buoyancy and diffusion important physical phenomena in combustion.
Important beginnings in the modeling of turbulent flows show promise as methods for representing the effects of energy release in the reacting, turbulent flows of combustors. Within the next decade, we can expect to see a significant impact of these modeling developments in the design procedures for real combustors.

\section{The Turbine Early Design Methods}

The first aircraft gas turbines were designed blade-row by blade-row. 10 This aerodynamic design process was similar to the early blade element methods used in the design of axial flow compressors. The main emphasis was selection of the velocity diagrams entering and leaving the blade row and the use of empirical loss data and flow blockage factors. Generally the endwalls were assumed cylindrical, although conical configurations were considered for some multistage applications. Inter-blade-row velocity diagrams were calculated assuming simple radial equilibrium and in most cases free vortex distribution of the whirl component. Blade-to-blade velocity distributions were then calculated with a simple twodimensional velocity gradient (stream filament) approach with assumed variations in total pressure loss and the mass flow parameter from the blade inlet plane to the blade exit plane. These calculations were used iteratively with assumed blade profiles until aerodynamically satisfactory shapes were evolved.

The gas temperatures and pressures were low enough in the early gas turbines that uncooled blades were adequate. As the cycle pressure ratios and turbine inlet temperatures increased, cooled turbine blading became a requirement. The gas-side heat transfer estimates for the turbine were based on empirical turbulent flow correlations for the flat plate representation of the blade row. Inside the blade or vane coolant passages one-dimensional channel or pipe flow correlations were employed in estimating the heat transfer coefficient. By iterating between the gas-side and coolant-side calculations, it was possible to estimate a wall temperature at a specified blade or vane location. This early heat transfer approach failed to comprehend the complexity of the convection mechanisms and the intricate geometry of the blades or vanes. It was apparent in this time period that more sophisticated design procedures were needed for the heat transfer and aerodynamics of the turbine. Research programs were initiated to correct this deficiency. A comprehensive picture of the development of the turbine design process during the $50^{\prime} s$ and 60 's and into the early 1970 decade is documented.11 This three-volume set was compiled by the staff of the Turbine Branch at the Lewis Research Center to serve as a turbine design handbook.

\section{Current Design Methods}

Currently, quasi-three-dimensional inviscid flow analyses are standard design tools for flow analysis and these are used with empirical and calculated boundary layer and endwall effects. The more modern design approaches place great reliance on knowledge about the entire flow path in the turbine blade passage. 12 Viscous secondary flows and such boundary layer phenomena as 
transition are considered in this design methodology. The blade aerodynamic geometry of advanced turbines is becoming more complex to allow for high loading distributions over the span of the blades. As a further consequence of this geometric complexity and higher loadings, the secondary flows are an increasingly important part of the overall flow pattern.

In addition to the turbine aerodynamics, current thermal design procedure involves more advanced heat transfer predictions to define more precisely the metal temperatures of the components. Hot gas-side heat transfer calculation methods range from the use of flat plate heat transfer correlations to fairly sophisticated boundary layer codes with various turbulence models, transition criteria, and provisions for geometric shapes such as curvature. Internal flow and heat transfer calculations for coolant side heat transfer typically use one-dimensional convection models with empirical correlations for impingement, pin fins, and turbulence promoters. Metal temperature prediction systems use these heat transfer calculations with three-dimensional conduction codes to estimate the gas-side and coolant side wall temperatures along the blade profile at several spanwise positions. The calculation is performed iteratively until prescribed wall temperatures are met through adjustment of the coolant-side conditions.

While currently used design methods provide turbines with high aerodynamic efficiencies, substantial uncertainties continue in the prediction of local metal temperatures. An analysis of the ability to predict turbine metal temperatures is discussed. 13 Figure 6 depicts a learning curve in turbine design technology and also shows an assessment of the current ability to predict metal temperatures. Note that gas-side heat transfer coefficient uncertainty is 35 percent while the coolant side uncertainty is 25 percent. These uncertainties, combined with only approximate knowledge of gas and coolant temperature, lead to an uncertainty of about $100 \mathrm{~K}\left(180^{\circ} \mathrm{F}\right)$ in local metal temperature for the operating conditions of current engines. This in turn leads to uncertainty in life prediction of a factor of ten. It should be noted that a significant contributor to this limitation on metal temperature prediction ability is the lack of precise knowledge of the real engine environment. There is a need for accurate measurements of temperature, pressure, and turbulence of the gases entering the turbine. The price paid for this uncertainty in metal temperature prediction can be quite high in terms of development cost as well as subsequent maintenance costs. Figure 7 shows a pie chart on the cost of development of a new engine from information supplied by several of the gas turbine engine companies. This representation averages the response. The total engine development costs in 1979 ranged from $\$ 500$ million to $\$ 1.2$ billion. In 1985 this would scale up to at least $\$ 600$ million to $\$ 1.6$ billion. Of that total cost, 10 to 40 percent were incurred in the core turbine. And two thirds of that was in fixes. That amounts to about $\$ 50$ to $\$ 500$ million in core turbine changes through flight certification and perhaps one year of operating experience. Recent conversations with, and research proposals from, the engine companies indicate that this picture has not significantly changed.

\section{Future Design Codes}

Current efforts in computational fluid mechanics, instrumentation, and computer technology hold promise of another period of rapid advancement in turbine design technology. We won't see dramatic increases in turbine inlet temperature such as the $550 \mathrm{~K}\left(1000^{\circ} \mathrm{F}\right)$ increase in the 50 's and 60 's, but we will see greatly increased computer involvement in design optimization and the simulation of component and full engine operation. For the aerodynamic and heat transfer codes, we will see significantly more accurate definition of boundary conditions because of improved high temperature instrumentation and greatly improved modeling of combustors. Threedimensional viscous computer codes will be standard design tools, and the uncertainty margins in heat transfer coefficients will be reduced by a factor of three. This, in combination with accurate knowledge of the environment, should permit metal temperature prediction with an accuracy of 14 to $28 \mathrm{~K}\left(25\right.$ to $\left.50^{\circ} \mathrm{F}\right)$ and greatly reduce the time and cost of engine development.

Improved aerodynamic and thermodynamic turbine design tools are needed to decrease the life cycle costs of operating engines. In addition to reducing development costs, these design methods will lead to increased hot part life, and reduced fuel consumption. There has been an awareness of these specific needs for many years as incremental improvements in design techniques were continually developed. Future improvements will depend upon improved instrumentation, new and improved computer codes, and the continuing improvement in computers. Two-dimensional codes for annular ducts and turbomachinery blade-to-blade flow surfaces are already in wide spread use, and at least one three-dimensional code has been used with some success by one of the engine companies. Many individuals and organizations are working on diverse approaches to the three-dimensional problem. These codes, with various levels of sophistication will be integrated into the design system. An inviscid three dimensional code presented ${ }^{14}$ has been tested against experimental blade passage flow measurements in an annular cascade. 15 Generally the inviscid three dimensional code predicted the features of the flow very well and is definitely more accurate than the two dimensional codes used in turbine design. Figure 8 outlines the features that will be incorporated into the advance design codes. In addition to those listed, unsteady flow effects must be handled in some way. The other needs have already been discussed to some extent, and certainly must be included in this list. The parameter $A N^{2}$ is commonly used to identify blade root centrifugal stress level. This product of annular flow area and the square of rotational speed is directly proportional to blade centrifugal stress given a material density and a taper factor. Increases in currently limiting $\mathrm{AN}^{2}$ values are desired by all aircraft turbine designers for the improvement of aerodynamic performance. Improved materials and cooling are the keys to improvement here.

\section{Concluding Remarks}

Judging from current trends, the design of the principal components of a gas turbine engine is transitioning from an empirical, trial and 
error process to a more computationally-based process. Computational codes which can describe steady, three dimensional fluid flow and heat transfer are becoming operational. Codes which will comprehend unsteadiness are being thought of, but in 1984 nothing is availabile which includes three dimensions along with the complication of unsteadiness. The ultimate in the design codes must include both of these. The type and characteristics of the codes that will be used widely in the future cannot be predicted. Just a few years ago it appeared that implicit type calculations (solving a set of differential equations at each step) appeared highly promising because of reduced computer time and inherent stability. However, the introduction of the vectorized computers has made the explicit methods (solve the unknowns one at a time at each step) appear attractive. From conversations with computational experts at the Lewis Research Center, it appears that the architecture of future computers will be the principal deterministic factor affecting the overall character of the codes. The most successful and powerful codes will make optimum use of computer architecture. Former debates about implicit and explicit coding may not be too relevant.

The output information from computational fluid mechanics and heat transfer codes will be part of the input to the structural codes that will predict the life and durability of the engine components. In current design practice, the prediction of structural lifetimes is highly uncertain because of a combination of inaccuracies in the prediction of local parameters such as temperature and pressure. Future codes are expected to bring about major improvements in the precision of estimating component life.

The new code predictions will have to undergo rigorous verifications before they can be accepted. Experiments will be devised to validate and certify the accuracy of the analyses and the appropriateness of the physical modeling. Judging from recent experience in verification of codes, this will be a major effort. However, the rewards from the availability of comprehensive design codes for the compressor, combustor, turbine and other components not discussed make the overall effort to develop these codes very worthwhile. The saving of time and expense in the development of future gas turbine engines will justify this effort. These engines should be more durable and reliable than their predecessors.

More specifically, we can expect to see some of the following improvements in the major components of future engines. The compressors will exhibit higher pressure ratios per stage and will be less susceptable to stall and surge over their range of operation. Structurally, the blades will be less likely to encounter flutter and tuned vibrations.

The combustors will be capable of operation at higher pressures and outlet gas temperatures. More uniform temperature pattern factors will be realized at the combustor exit. The future combustor will be more tolerant of the varying specification of fuels including synthetic types.
These benefits will accrue because of:

1. improved predictability of fuel sprays,

2. models of combustion reactions in a gas stream

3. three dimensional models of mixing.

The turbines will be able to accept higher enthalpy gas. Despite the more severe thermal conditions of the working gas, more efficient use will be made of the cooling air bled from the compressor. Tip clearance losses will be minimized. Each stage of the turbine will operate at a higher work factor and the parasitic losses will be minimal. The more accurate prediction of wall temperatures from codes will result in more reliable life predictions. This advance should mitigate the turbine maintenance uncertainty which troubles the airlines in current operations.

The greater capability to predict the operating conditions throughout the engine will encourage designers to make bolder changes between engine designs. Innovation rather than just extrapolation will be practiced in proposing new designs.

Al1 in a11, the computational design methodology will introduce a new era into the aviation gas turbine industry. In the international competition for markets, the companies which introduce reliable, high performarce gas turbine engines will thrive. They will be the companies that have adopted and implemented sophisticated computational methods into the design procedure.

\section{REFERENCES}

\section{Compressor Section}

1. Johnsen, I.A., and Bullock, R.0., "Aerodynamic Design of Axial Flow Compressors," NASA SP-36, 1965.

2. Wu, Chunq-Hua, "A General Theory of ThreeDimensional Flow in Subsonic and Supersonic Turbomachines of Axial-, Radial-, and MixedFlow Types," NACA TN-2604, 1952.

Analytical Approach for Multistage Turbomachinery Section

3. Adamczyk, J.J., "A Model Equation for Simulating Flows in Multistage Turbomachinery." Proposed TM.

4. Adkins, G.G., and Smith, L.H., "Spanwise Mixing in Axial-Flow Turbomachines," ASME Paper 81-GT-57, American Society of Mechanical Engineers, 1981.

The Combustor Design Practice

5. Lefebrre, A.H., "Gas Turbine Combustion," McGraw-Hill, New York, 1983.

6. "Basic Considerations in the Combustion of Hydrocarbon Fuels with Air," NACA Report $1300,1959$. 
7. Norgren, C.T., and Childs, H.H., "Effect of L.iner Air-Entry Holes, Full State, and Combustor Size on Performance of an Annular Turbojet Combustor at Low Pressures and High Air-Flow Rates," NACA RM E52J09, Jan. 1953.

8. Ghoniem, A.F., Marek, C.J., and Oppenheim, A.K., "Modeling Interface Motion of Combustion (MIMOC) - A Computer Code for Two-Dimensional, Unsteady Turbulent Combustion," NASA TP-2132, August 1983.

9. Boris, J.P., Oran, E.S., Fritts, M.L. and Oswald C., "Time Dependent, Compressible Simulations of Shear Flows: Tests of Outflow Boundary Conditions," NRL-MR-5249, Naval Research Laboratory, Dec. 1983.

Future Turbine Design Codes

10. Stewart, W.L., "Analytical Investigation of Multistage-Turbine Efficiency Characteristics in Terms of Work and Speed Requirements, "NACA RM-E-57K22b, Feb. 1958.

11. Glassman, A.J. ed., "Turbine Design and Application," NASA SP-290-Vol. 1, 1972; -Vol. 2, $1973 ;-V_{0} 1.3,1975$.
12. Boyle, R.J., Haas, J.E., and Katsanis, T. "Comparison Between Measured Turbine Stage Performance and the Predicted Performance Using Quasi-3D Flow and Boundary Layer Analyses," NASA TM-83640, June 1984.

13. Stepka, F.S., "Uncertainties in Predicting Turbine Blade Metal Temperatures," ASME Paper 80-HT-25, American Society of Mechanical Engineers, Ju1y 1980.

14. Denton, J.D., and Singh, U.K., "Time Marching Methods for Turbomachinery Flow Calculation," Application of Numerical Methods to Flow Calculations in Turbomachines. Von Karman Institute for Fluid Dynamics Lecture Series 1979-7, 1979.

15. Goldman, L.J., and Seasholtz, R.G., "Comparison of Laser Anemometer Measurements and Theory in an Annular Turbine Cascade With Experimental Accuracy Determined by Parameter Estimation," NASA TM-82860, Nov. 1982. 


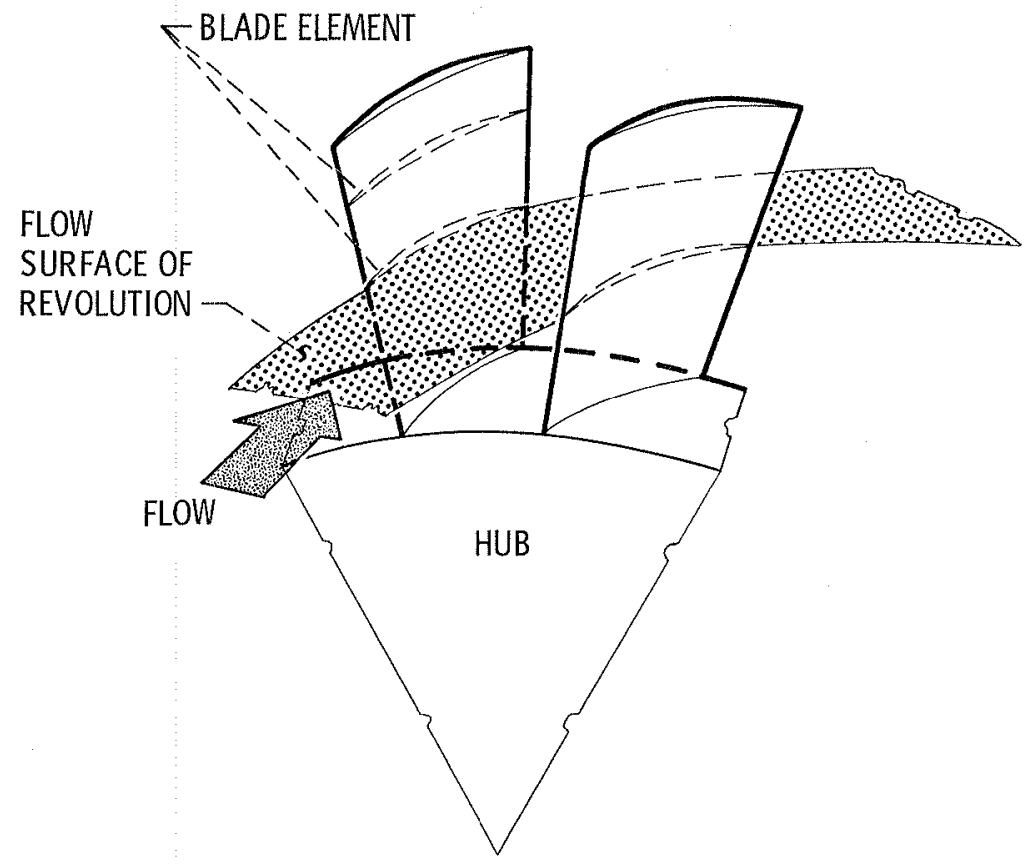

Figure 1. - Compressor blade element design approach.
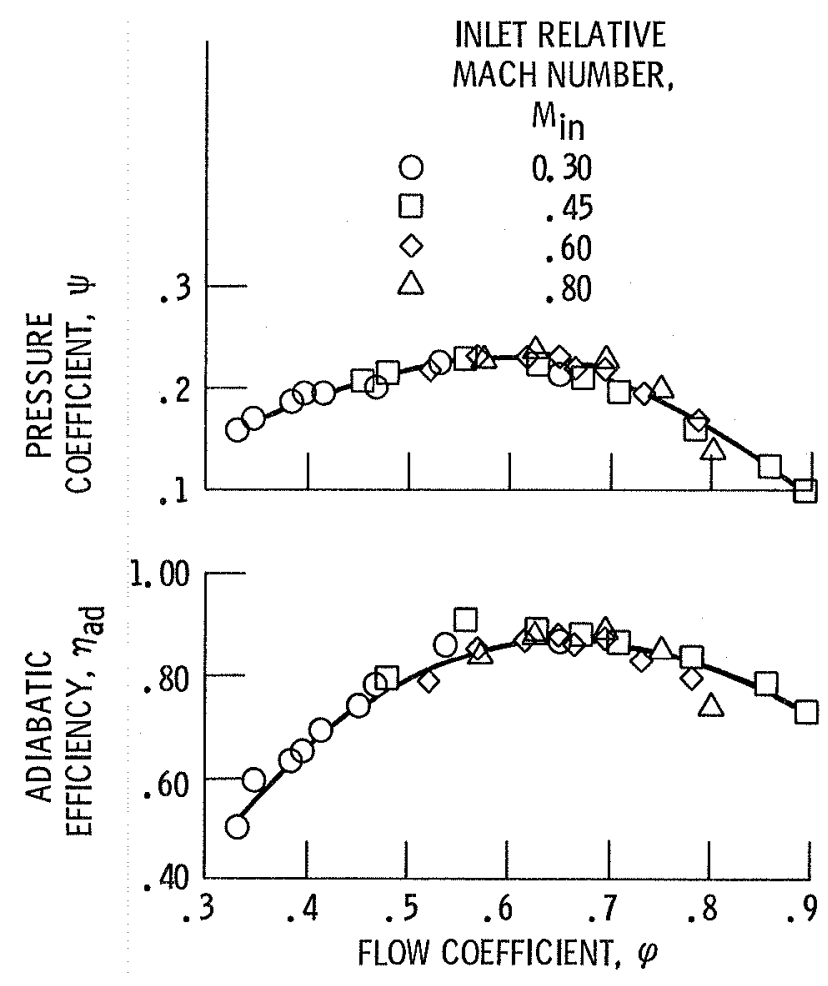

Figure 2. - Typical compressor dimensionless performance map. 


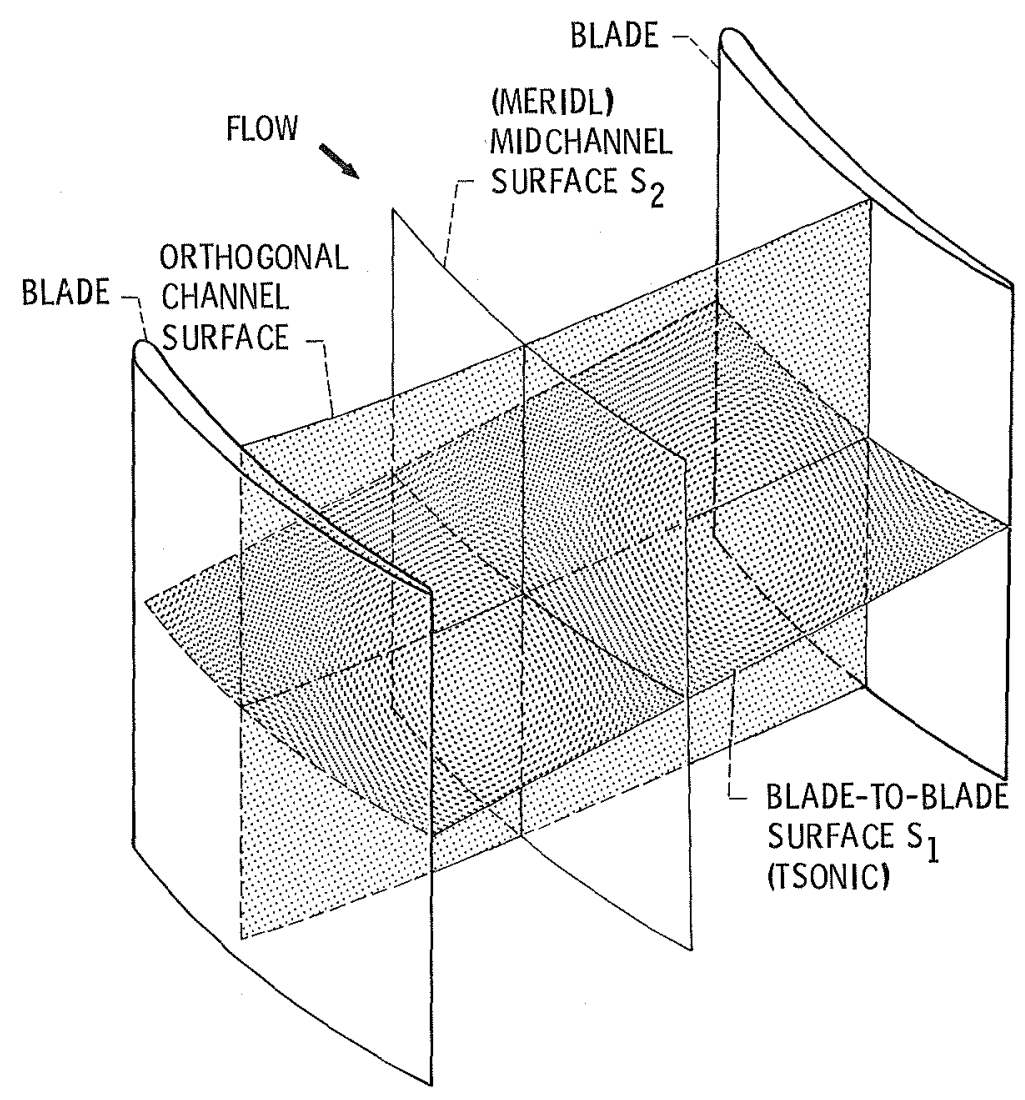

Figure 3. - Analysis surfaces in a turbomachine.

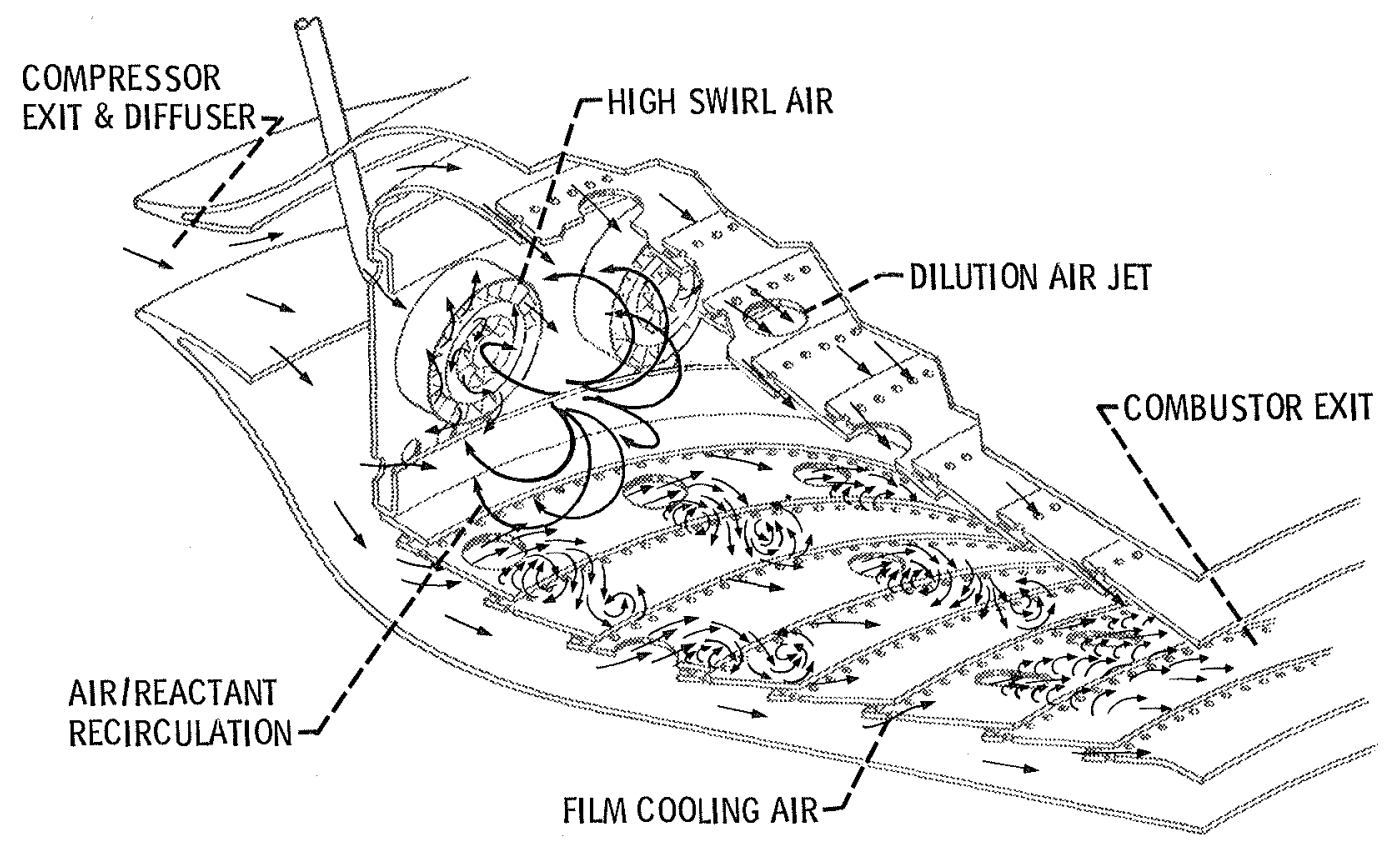

- FULLY 3-DIMENSIONAL FLOW

- CHEMICAL REACTION/HEAT RELEASE

- HIGH TURBULENCE LEVELS

- 2 PHASE WITH VAPORIZATION

Figure 4. - Combustor flow phenomena. 


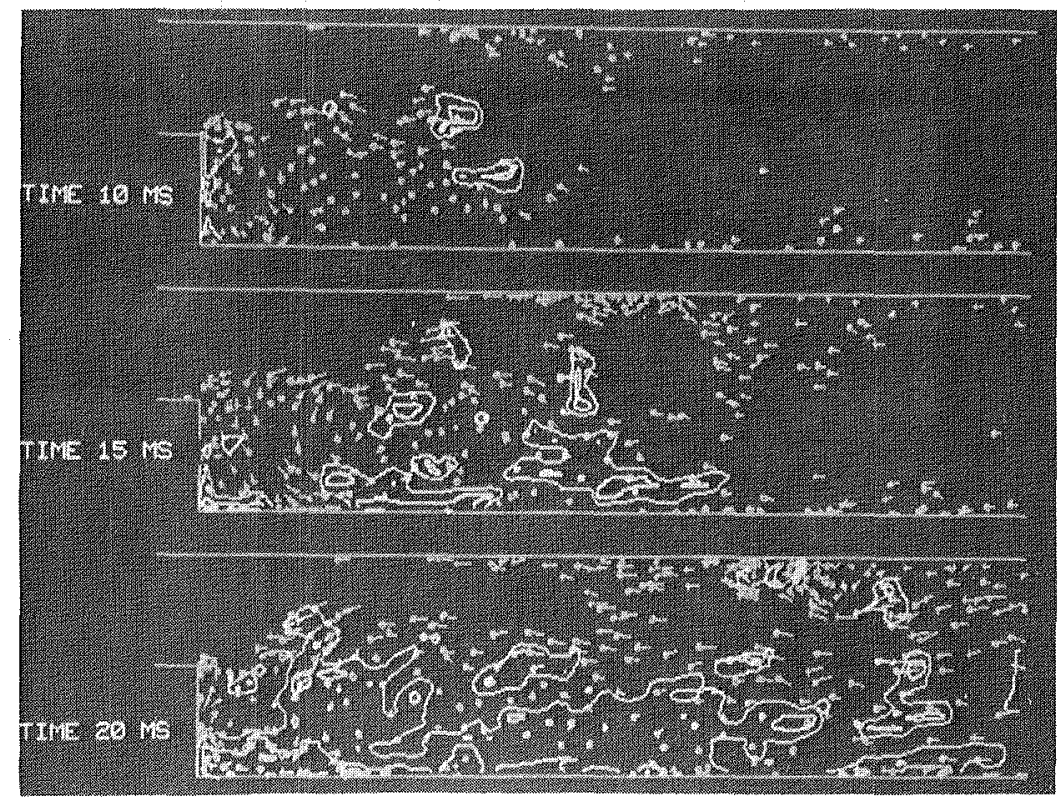

Figure 5. - Computational graphical representation of combustion process downstream of a 2D step. Three time intervals.

CD-83-13778

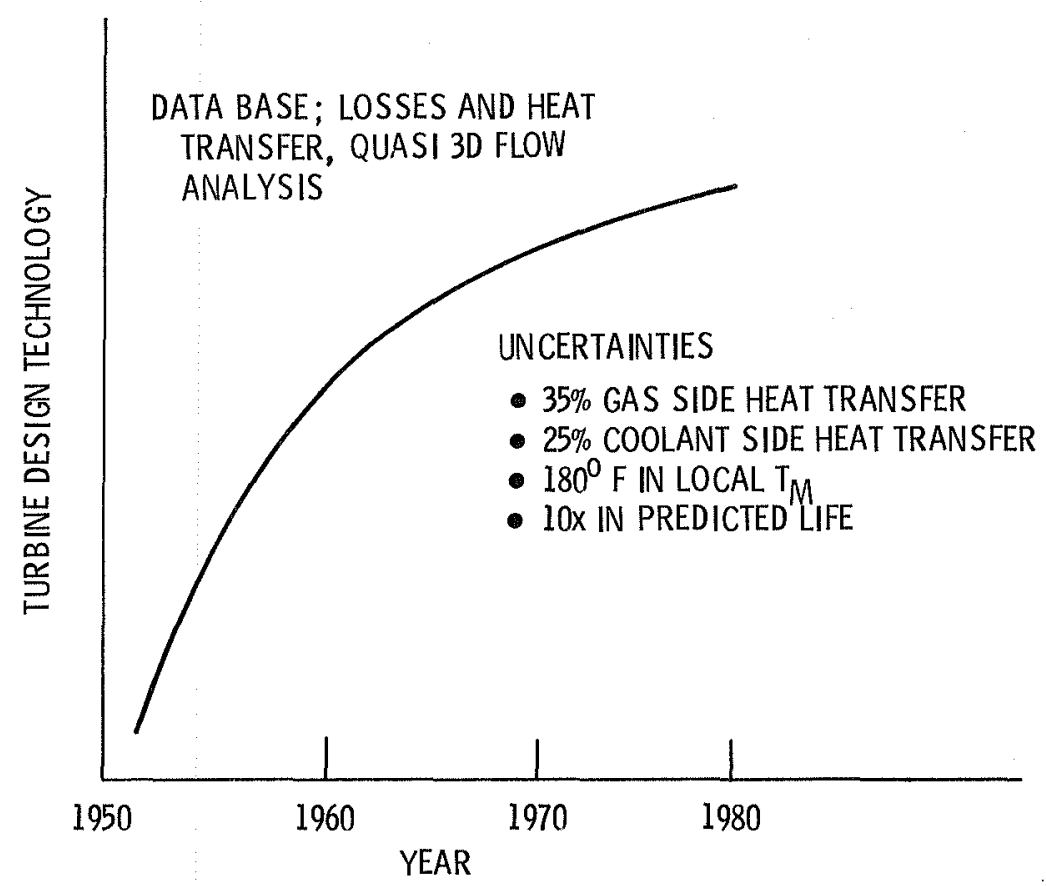

Figure 6. - High pressure turbine technology advance since 1950. 


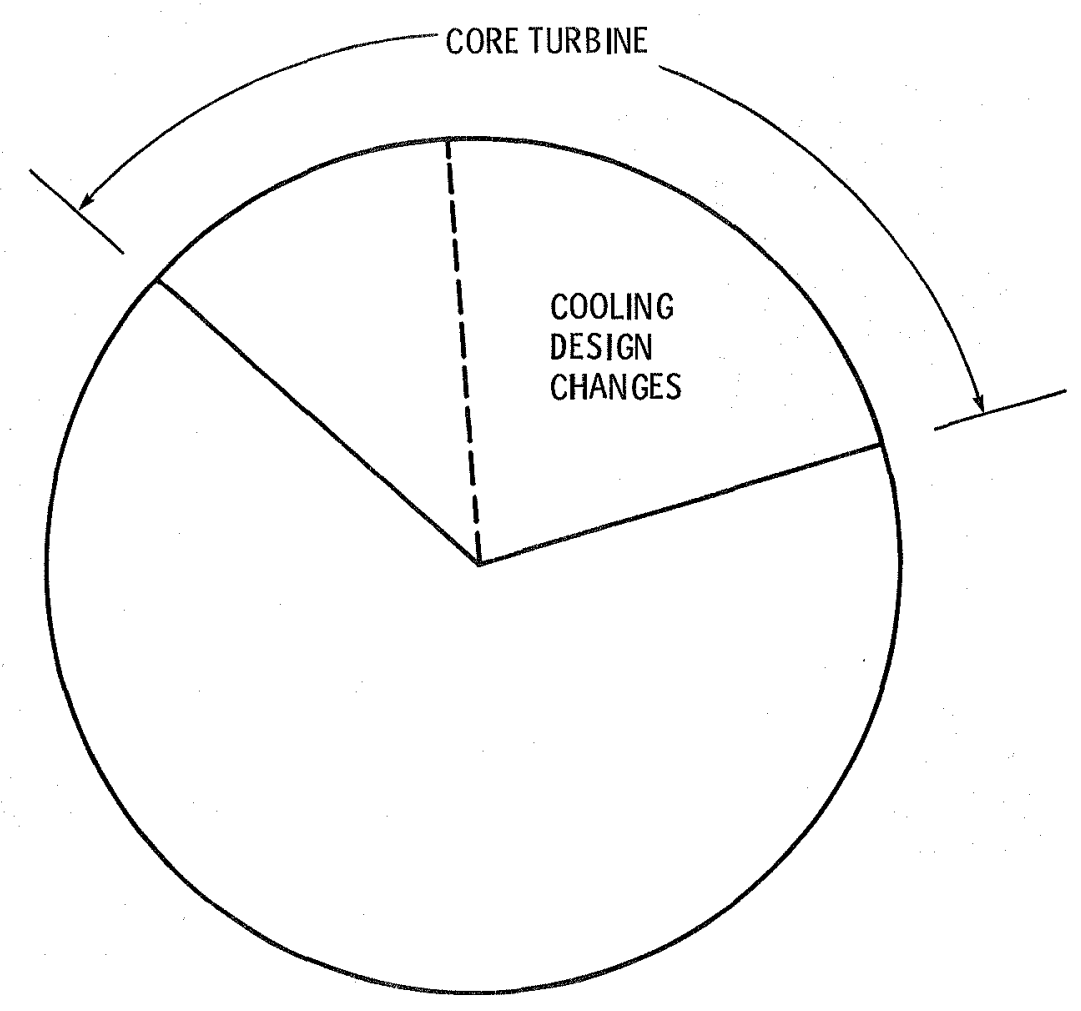

• $600 \mathrm{~m}$ T0 $1600 \mathrm{~m} \$$

- 30 TO 40\% IN CORE TURBINE

-2/3 OF THIS IN "FIXES"

Figure 7. - New engine development cost,

-3D VISCOUS FLOW CODES

- LEADING EDGES

- TRAILING EDGES

- ROTATION

- HEAT TRANSFER

- COOLANT ADDITION

- FLOW AND HEAT TRANSFER CODES FOR LOCAL AREAS

- COOLING HOLES AND SLOTS

- COOLANT PASSAGES WITHIN VANES AND BLADES

- FLOW AND HEAT TRANSFER CODES FOR DISK CAVITIES

-WELL DEFINED TURBINE ENVIRONMENT

- TEMPERATURE AND PRESSURE PROFILES

- TURBULENCE \& UNSTEADINESS

- COOLANT FLOW CONDITIONS AND DISTRIBUTIONS

- REDUCED TIP CLEARANCES AND SENSITIVITY

- INCREASED AN ${ }^{2}$ (PRODUCT OF ANNULAR FLOW AREA \& (SPEED) ${ }^{2}$ )

Figure 8. - Long range needs in turbine design technology. 


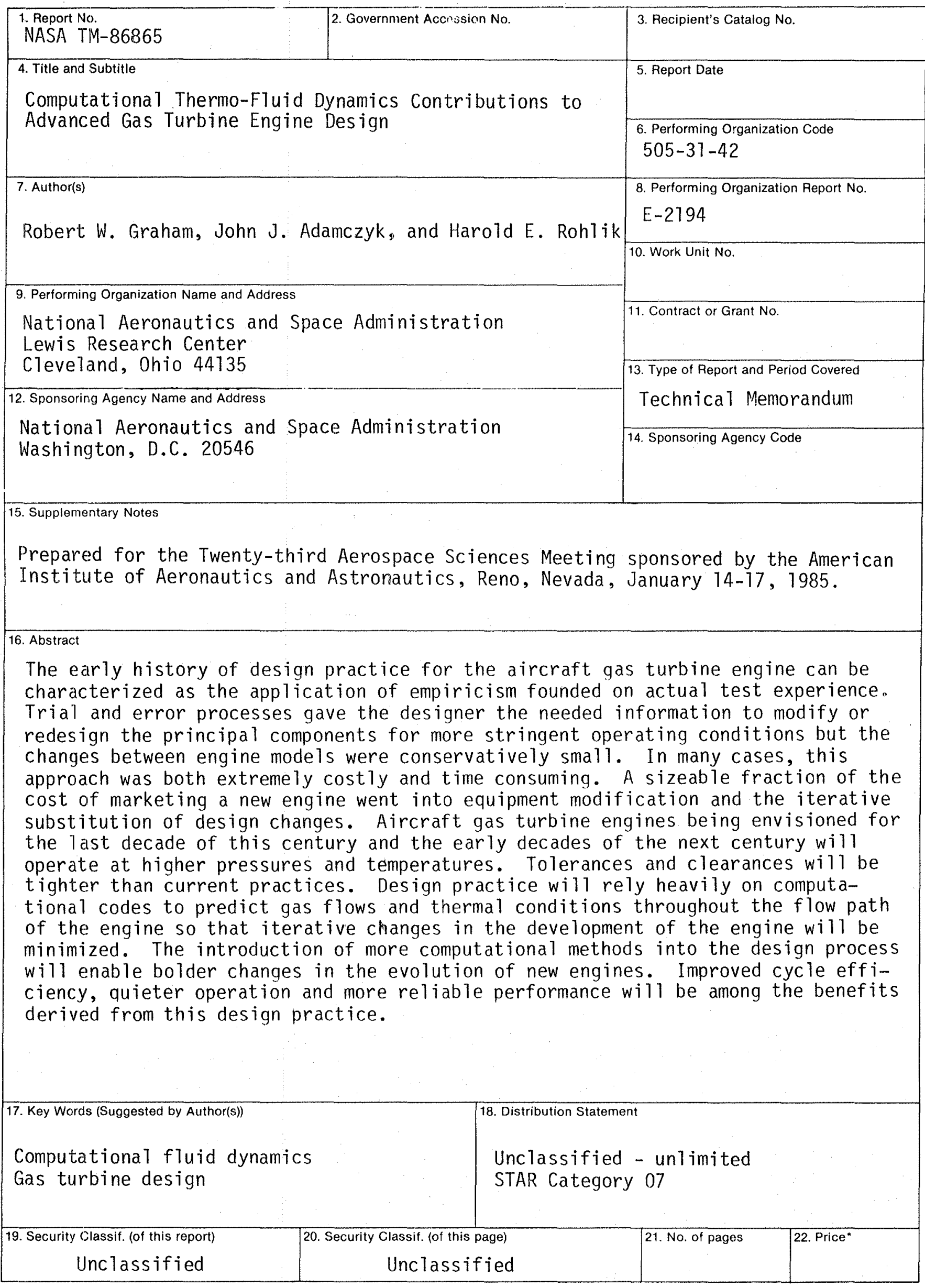

*For sale by the National Technical Information Service, Springfield, Virginia 22161 


\section{End of Document}

